\title{
Heart Rate Variability as an Early Objective Indicator of Subjective Feeling of Depression in Daily Life
}

\author{
Han-Gue Jo ${ }^{1}$, Nara Shin ${ }^{2}$ \\ ${ }^{1}$ School of Computer Information and Communication Engineering, Kunsan National University, Gunsan, Korea \\ ${ }^{2}$ Human Rights Center, Konkuk University, Seoul, Korea
}

Objectives: Changes in the autonomic nervous system have been observed in patients with depressive disorders by measuring their heart rate variability (HRV). However, whether HRV associates with depressive events in healthy people remains unknown. Methods: Four healthy people participated in the present study. Their HRVs were measured routinely for 6 to 13 months. During this time, two participants reported experiencing two and three bouts of depression, respectively. This approach allowed us to examine changes in the participants' HRVs by comparing their HRVs from before and after the unexpected depressive events. Changes in HRV were compared against those of two participants who did not report any depressive event. Results: Participants' low frequency/high frequency (LF/HF) ratios of HRV were lower after the event of depression than before. Their LF/HF ratios increased after recovery from the depressive events. In contrast, two participants who did not report any depressive event showed relatively smaller changes in their LF/HF ratios across measurements. Conclusions: These results suggest that the LF/HF ratio may provide an objective measure of subjective experiences of depression and help identify potential cases of clinical depression.

Keywords: Depression, Autonomic Nervous System, Electrocardiogram, Heart Rate, Individuality

Submitted: December 6, 2020

Revised: 1st, December 16, 2020; 2nd, May 4, 2021

Accepted: May 28, 2021

\section{Corresponding Author}

Han-Gue Jo

School of Computer Information and Communication Engineering, Kunsan National University, 290-2, Miryong-dong, Gunsan 54150, Korea. Tel: +82-63-469-4694, E-mail: hgjo@kunsan.ac.kr (https:// orcid.org/0000-0003-0595-675X)

This is an Open Access article distributed under the terms of the Creative Commons Attribution Non-Commercial License (http://creativecommons.org/licenses/by$\mathrm{nc} / 4.0 /$ ) which permits unrestricted non-commercial use, distribution, and reproduction in any medium, provided the original work is properly cited.

(c) 2021 The Korean Society of Medical Informatics

\section{Introduction}

A depressed mood can occur unexpectedly in one's daily life, and repeated bouts of depression can develop into clinical depression. The subjective severity and frequency of depressive symptoms vary from person to person and can be experienced differently. An objective measure for determining the severity of depression could help detect depressive symptoms before they develop into clinical depression.

Many studies have attempted to identify a link between depression and the autonomic nervous system (ANS) measured using heart rate variability (HRV) (See [1]). HRV is a measure of the interval between heartbeats, and it has been suggested that the frequency component of HRV may reflect the state of one's ANS (See [2,3]). The high-frequency (HF) component of HRV measures the activity of the car- 
diac branches of the vagus nerve, which is known to indicate parasympathetic nerve activity. In contrast, the lowfrequency (LF) component indicates both parasympathetic and sympathetic nerve activity. Therefore, the LF/HF ratio indicates the degree of balance between the sympathetic and parasympathetic nervous systems. Two meta-analyses found that patients with depressive disorders had reduced LF and HF components and a higher LF/HF ratio than the healthy control group $[4,5]$. However, not all studies support this finding. Some studies found no differences in HRV indices between patients with depressive disorder and control groups, and evidence of heterogeneity was found in clinical studies [6-10]. Most of those studies investigated HRV indices of patients with major depressive disorder and compared with a healthy control group or examined patients' HRVs after a clinical diagnosis had been made [11]. However, to the best of our knowledge, no studies have examined whether HRV associates with unexpected depressive events in healthy people.

Two healthy volunteers who had no symptoms of clinical depression participated the present study. HRV measurements were taken nine times for one participant and 16 times for the other participant across several months, during which time the participants reported experiencing three and two unanticipated events of depression, respectively. Since the participants were healthy and showed no prior symptoms of clinical depression, we were able to examine changes in HRV before and after the unexpected depressive event on an individual basis. It was hypothesized that changes in the participants' LF/HF ratios would be recorded after the depressive events.

\section{Methods}

Two women participated in the present study (Table 1). After resting in a seated position for more than 10 minutes, the participants' HRV was measured using a computerized three-channel digital electrocardiography system (model QECG-3; Laxtha Inc., Daejeon, Korea) with bipolar limb leads, recording for 310 seconds with a sampling rate of $256 \mathrm{~Hz}$ [12]. HRV was measured at a particular time of day, between 19:00 and 20:00. Participants were instructed to abstain from smoking, caffeinated beverages, alcohol, and heavy exercise for 12 hours before measurements were taken. The measurements were taken in a quiet laboratory room. In addition, the participants' self-report ratings of mood were estimated using the Beck Depression Inventory II (BDI-II) [13] and the Beck Anxiety Inventory (BAI) [14]. The Institutional Review Board of Kongju National University approved the present study, and informed consent was obtained from all participants.

The HRV of participant 1 (P1) was measured 16 times with intervals of at least 1 week. The average interval between measurements was 3.5 weeks. During this time, the participant reported experiencing two bouts of depression caused by interpersonal relationship conflicts. The first incident occurred before the fifth HRV measurement, and the second incident occurred before the sixth measurement. After the second incident, P1 was admitted to a psychiatric hospital and received on-site treatment. Medication was prescribed before the seventh measurement (two daily doses taken 30 minutes after breakfast and dinner). The participant reduced the dosage to one daily dose since she felt better after the eighth measurement. The participant stopped taking the medication around the ninth measurement due to drowsiness. The participant was then prescribed a second medication regimen to be taken after dinner, however, the medication was taken irregularly. The first medication regimen consisted of a combination of Newpram (escitalopram oxalate, 5 mg tablets; Myung In Pharm Co. Ltd., Seoul, Korea), Indenol (propranolol hydrochloride, $10 \mathrm{mg}$ tablets; Dongkwang Pharm Co. Ltd., Seoul, Korea), and Alpram (0.125 mg tablets; Whanin Pharm Co. Ltd., Seoul, Korea). The second regimen consisted of Newpram (escitalopram oxalate, $10 \mathrm{mg}$

Table 1. Characteristics of participants

\begin{tabular}{|c|c|c|c|c|c|c|c|}
\hline & Sex & Age (yr) & Height (cm) & Weight (kg) & BMI $\left(\mathrm{kg} / \mathrm{m}^{2}\right)$ & SMM (kg) & $\begin{array}{l}\text { Number of HRV } \\
\text { measurements }\end{array}$ \\
\hline $\mathrm{P} 1$ & Female & 25 & 164.2 & 54.3 & 20.1 & 19.7 & 16 \\
\hline $\mathrm{P} 2$ & Female & 23 & 167.3 & 67.3 & 24.0 & 22.4 & 9 \\
\hline P3 & Female & 22 & 157.6 & 41.4 & 16.7 & 17.1 & 13 \\
\hline $\mathrm{P} 4$ & Male & 25 & 160.0 & 61.4 & 24.0 & 44.8 & 10 \\
\hline
\end{tabular}

During the present study, P1 and P3 did not engage in physical exercise, P2 swam regularly, and P4 played soccer weekly. BMI: body mass index, SMM: skeletal muscle mass. 
tablets), Indenol (propranolol hydrochloride, $10 \mathrm{mg}$ tablets), and Alpram (0.125 mg tablets). The subjects' scores for depression and anxiety measured by the BDI-II and BAI were assessed after the sixth HRV measurement were 18 points (14-19 indicates mild depression) and 14 points (8-15 indicates mild anxiety), respectively.

The HRV of participant 2 (P2) was measured nine times across 6 months with intervals of at least 1 week. The average interval between measurements was 2.5 weeks. The participant reported experiencing depression three times due to deadlines for work assignments. The participant reported that these bouts of depression were temporary. The participants' BDI-II and BAI scores were measured after the second measurement, during which the first depressive event was reported, were 4 points ( $0-9$ indicates minimal depression) and 6 points ( $0-9$ indicates normal or no anxiety), respectively.

The time and frequency domains of the HRV data were analyzed, which included the standard deviation of NN intervals (SDNN), the percentage of successive RR intervals that differed by more than $50 \mathrm{~ms}$ (pNN50), root mean square of successive differences between adjacent RR intervals (RMS$\mathrm{SD}$ ), total power (TP; 0-0.4 Hz), very-low-frequency power (VLF; 0-0.04 Hz), low-frequency power (LF; 0.04-0.15 Hz), high-frequency power (HF; $0.15-0.4 \mathrm{~Hz}$ ), and the LF/HF ratio (ratio of LF to HF power).
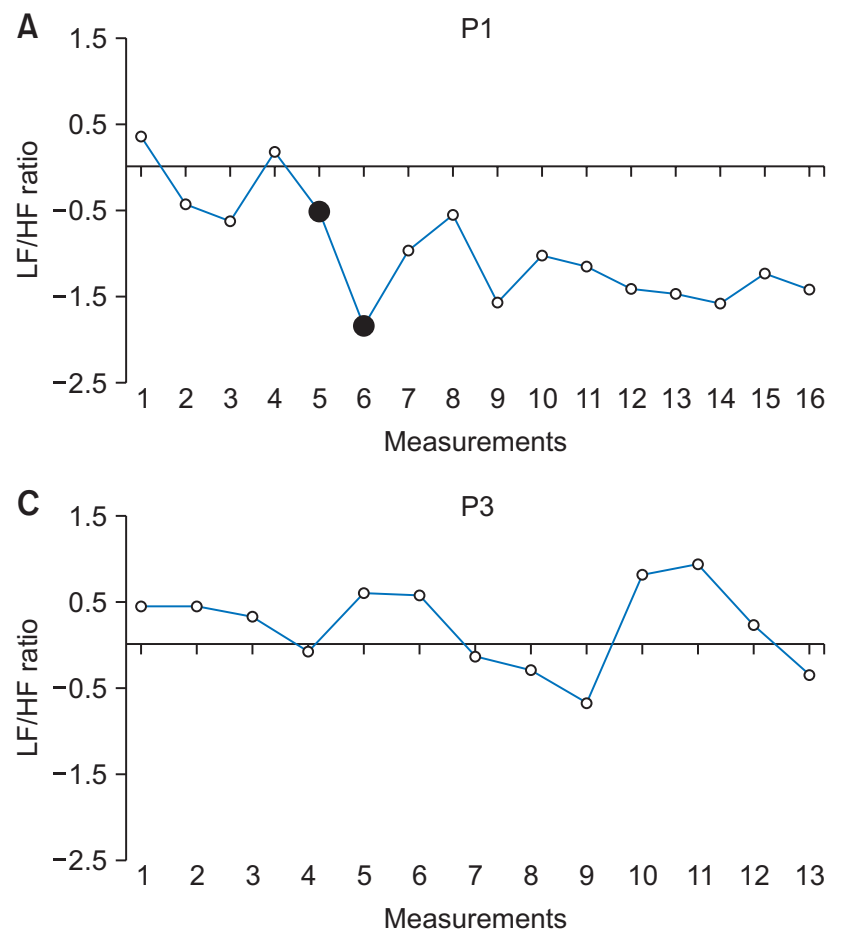

Figure 1. Changes in the LF/HF ratio in four participants: (A) P1, (B) P2, (C) P3, (D) P4. Black dots indicate when the participants reported having experienced depression. LF: low frequency power, HF: high frequency power.
In addition, HRV data were obtained from two healthy participants (participants 3 and 4; P3 and P4 in Table 1) who did not report experiencing depressive event during the course of the study. The average measurement intervals were 1.1 weeks for $\mathrm{P} 3$ and 1.2 weeks for P4, respectively.

\section{Results}

The changes in the LF/HF ratio for P1 are shown in Figure 1A. The fifth and sixth measurements showed a decline in the LF/HF ratio, in which P1 reported experiencing depression caused by unexpected interpersonal conflicts. P1's LF/ HF ratio remained low (average LF/HF ratio for 7-16 measurements, -1.243), and did not recover to pre-depression levels (average LF/HF ratio for 1-4 measurements, $-0.137 ; p$ $=0.011$ two-tailed Student $t$-test). P1's LF/HF ratio increased temporarily to 0.977 and -0.550 at the seventh and eighth measurements, respectively, while the participant was taking medication. However, the ratio decreased again to -1.563 at the ninth measurement after the participant stopped taking medication. The averages of analyzed HRV indices before and after the two depressive experiences are shown in Table 2.

The changes in the LF/HF ratio of P2 are shown in Figure 1B. P2 reported experiencing depression caused by workrelated stress during the second, fifth, and seventh measure-
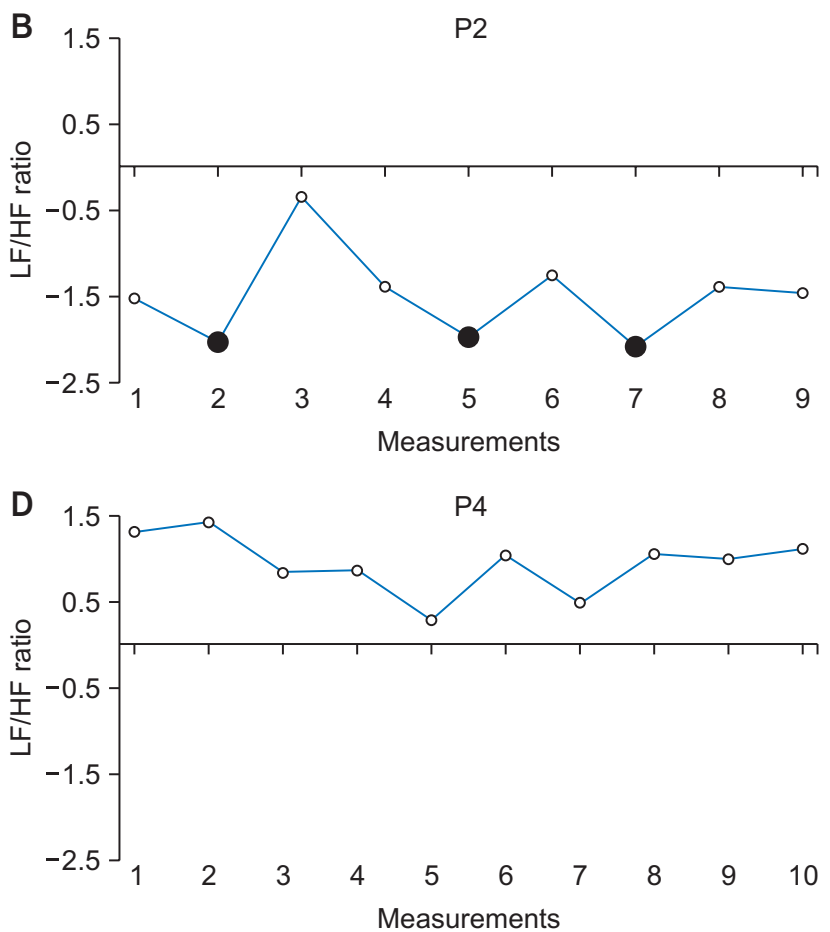


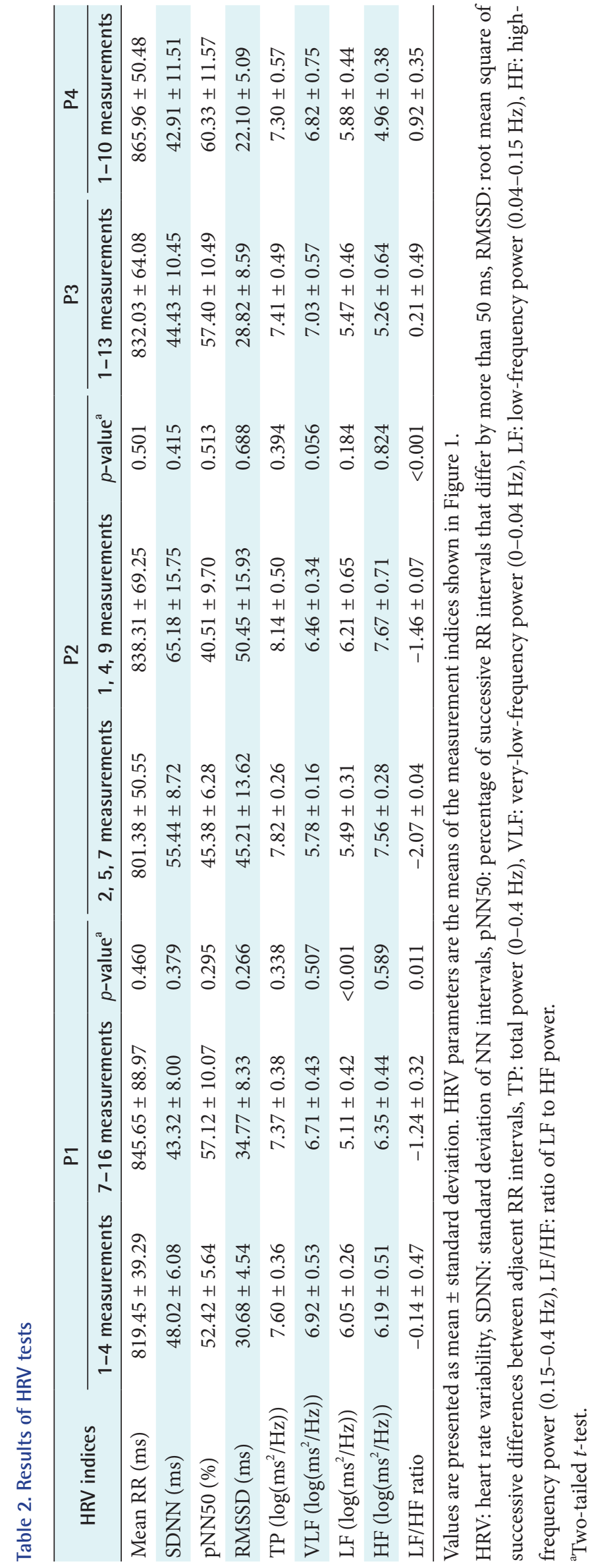


ments, after which relatively low LF/HF ratios were observed $(-2.074,-2.028$, and -2.107 , respectively). After P2 completed the work assignments, P2's LF/HF ratios increased to $0.347,-1.252$, and -1.384 for the third, sixth, and eighth measurements, respectively $(p<0.001$ two-tailed Student $t$ test) (see also Table 2).

The changes in $\mathrm{LF} / \mathrm{HF}$ ratios for $\mathrm{P} 3$ and $\mathrm{P} 4$, who reported no event of depression, are shown in Figure 1C and 1D, respectively. Smaller variations in LF/HF ratios were observed than in $\mathrm{P} 1$ and $\mathrm{P} 2$. The standard deviations of LF/HF ratios across measurements were 0.642 for $\mathrm{P} 1,0.547$ for $\mathrm{P} 2,0.489$ for $\mathrm{P} 3$, and 0.347 for $\mathrm{P} 4$, and the mean values of the $\mathrm{LF} / \mathrm{HF}$ ratios were -0.962 for $\mathrm{P} 1,-1.507$ for $\mathrm{P} 2,0.210$ for $\mathrm{P} 3$, and 0.920 for P4 (Table 2).

Exploratory analyses were performed to examine the relationships between individual bouts of depression and the other HRV indices in addition to the LF/HF ratio. However, no significant patterns were observed.

\section{Discussion}

The LF/HF ratio, which may indicate the balance of the ANS, decreased when participants reported experiencing depression caused by external stressors (P1 and P2), and increased after the psychological stressors were resolved, either due to completing stressful work assignments (P2) or taking medicine (P1). Such changes in LF/HF ratio were observed repeatedly in $\mathrm{P} 2$, suggesting a consistent pattern of decreases in the $\mathrm{LF} / \mathrm{HF}$ ratio corresponding to subjective experiences of depression. P1's LF/HF ratio continued to decrease at the fifth and sixth measurements, suggesting that the two successive interpersonal conflicts led to increases in depression severity. Indeed, P1 reported experiencing a severe depressive mood after the second interpersonal conflict, which occurred before the symptoms arising from the first conflict were not yet resolved. Conversely, variations in the LF/HF ratios of $\mathrm{P} 3$ and $\mathrm{P} 4$, who did not report any depressive event, were small compared to those observed in P1 and P2.

A reduced $\mathrm{LF} / \mathrm{HF}$ ratio is believed to indicate that parasympathetic nerve activity is more active than sympathetic nerve activity. Considering this context, increased parasympathetic nerve activity in P1 and P2 caused by bouts of depression in their daily lives may have led to ANS imbalances. This observation may contrast with previous findings, in which increases were observed in the LF/HF ratios of patients with depressive disorders [4,5]. Thus, it is plausible that the relationship between HRV and depression might differ between people with and without depressive disorders. Further stud- ies with a large sample size $(n=84$ in a matched-pairs design with an effect size of $0.4 ; \alpha=0.05$; and power of 0.95 ) are required to examine group effect on the experience of depression and its correlation with HRV.

The following limitations should be considered when interpreting the findings of this study. First, the findings are based on a case study of two healthy women in their twenties who experienced depression during the course of the study. The types of depressive symptoms and their severity were not examined, and participants' experience of depression were assessed subjectively. Therefore, the findings cannot be generalized to patients with depressive disorder. Second, the findings of this study may differ for men, younger adults, and older adults. The relationship between HRV and depression may be stronger for men than for women [15]. A metaanalysis of older adults found decreases in LF, but not in HF [10]. This previous finding contrasted to that of a meta-analysis of younger adults showing decreases in HF, but not in LF [4]. Therefore, future studies should consider the roles of sex and age in HRV. Third, we were not able to continuously monitor HRV. Since one's HRV varies over the course of the day, the present findings might have been affected by day-tonight variations observed in healthy people [16]. However, it should be noted that HRV of P1 and P2 were measured at the same time of day, between 19:00 and 20:00, to reduce the effect of daily variations in HRV. Lastly, although LF/HF ratio is known to correspond to the degree of autonomic nerve balance, it would help to verify the findings of this study to examine the associations between LF/HF ratio and various physical indicators such as respiration, electromyography, and electroencephalography.

Despite these limitations, this study observed changes in HRV indices before and after the onset of subjective feelings of depression in healthy adults. The bouts of depression in healthy individuals corresponded to a decrease in one's LF/ $\mathrm{HF}$ ratio. This finding suggests that $\mathrm{LF} / \mathrm{HF}$ ratio may be a biological substrate that links abnormal physiological function to depression. LF/HF ratio may provide an early objective indicator of subjective feelings of depression in daily life before the development of clinical depression.

\section{Conflict of Interest}

No potential conflict of interest relevant to this article was reported. 


\section{Acknowledgments}

The authors are grateful to Giho Jo who took part in planning and data acquisition.

\section{ORCID}

Han-Gue Jo (https://orcid.org/0000-0003-0595-675X)

Nara Shin (https://orcid.org/0000-0003-4540-3703)

\section{References}

1. Sgoifo A, Carnevali L, Alfonso Mde L, Amore M. Autonomic dysfunction and heart rate variability in depression. Stress 2015;18(3):343-52.

2. Berntson GG, Bigger JT Jr, Eckberg DL, Grossman P, Kaufmann PG, Malik M, et al. Heart rate variability: origins, methods, and interpretive caveats. Psychophysiology 1997;34(6):623-48.

3. Malliani A, Lombardi F, Pagani M. Power spectrum analysis of heart rate variability: a tool to explore neural regulatory mechanisms. Br Heart J 1994;71(1):1-2.

4. Kemp AH, Quintana DS, Gray MA, Felmingham KL, Brown K, Gatt JM. Impact of depression and antidepressant treatment on heart rate variability: a review and meta-analysis. Biol Psychiatry 2010;67(11):1067-74.

5. Koch C, Wilhelm M, Salzmann S, Rief W, Euteneuer F. A meta-analysis of heart rate variability in major depression. Psychol Med 2019;49(12):1948-57.

6. Yeragani VK, Pohl R, Balon R, Ramesh C, Glitz D, Jung I, et al. Heart rate variability in patients with major depression. Psychiatry Res 1991;37(1):35-46.

7. Liang CS, Lee JF, Chen CC, Chang YC. Reactive heart rate variability in male patients with first-episode major depressive disorder. Prog Neuropsychopharmacol Biol
Psychiatry 2015;56:52-7.

8. Licht CM, de Geus EJ, van Dyck R, Penninx BW. Association between anxiety disorders and heart rate variability in The Netherlands Study of Depression and Anxiety (NESDA). Psychosom Med 2009;71(5):508-18.

9. O'Regan C, Kenny RA, Cronin H, Finucane C, Kearney PM. Antidepressants strongly influence the relationship between depression and heart rate variability: findings from The Irish Longitudinal Study on Ageing (TILDA). Psychol Med 2015;45(3):623-36.

10. Brown L, Karmakar C, Gray R, Jindal R, Lim T, Bryant C. Heart rate variability alterations in late life depression: a meta-analysis. J Affect Disord 2018;235:456-66.

11. American Psychiatric Association. Diagnostic and statistical manual of mental disorders (DSM-5). Washington (DC): American Psychiatric Association; 2013.

12. Heart rate variability: standards of measurement, physiological interpretation and clinical use. Task Force of the European Society of Cardiology and the North American Society of Pacing and Electrophysiology. Circulation 1996;93(5):1043-65.

13. Beck AT, Ward CH, Mendelson M, Mock J, Erbaugh J. An inventory for measuring depression. Arch Gen Psychiatry 1961;4:561-71.

14. Beck AT, Epstein N, Brown G, Steer RA. An inventory for measuring clinical anxiety: psychometric properties. J Consult Clin Psychol 1988;56(6):893-7.

15. Chen HC, Yang CC, Kuo TB, Su TP, Chou P. Gender differences in the relationship between depression and cardiac autonomic function among community elderly. Int J Geriatr Psychiatry 2010;25(3):314-22.

16. Burger AJ, Charlamb M, Sherman HB. Circadian patterns of heart rate variability in normals, chronic stable angina and diabetes mellitus. Int J Cardiol 1999;71(1): 41-8. 